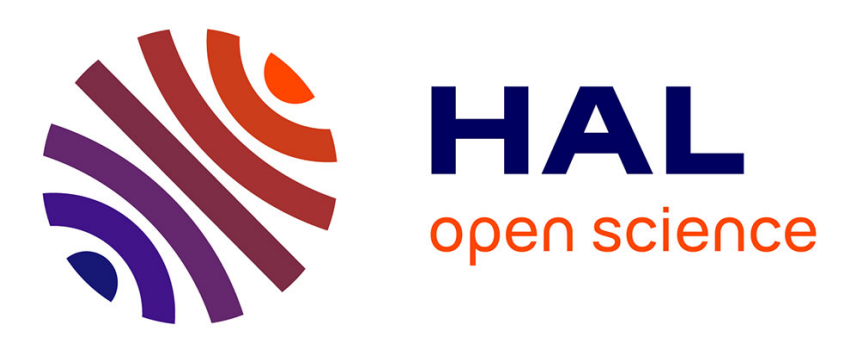

\title{
Robust asymptotic stabilization of nonlinear systems by state feedback
}

François Poulain, Laurent Praly

\section{To cite this version:}

François Poulain, Laurent Praly. Robust asymptotic stabilization of nonlinear systems by state feedback. 8th IFAC Symposium on Nonlinear Control Systems, Sep 2010, Bologna, Italy. pp.653-658. hal-00541541

HAL Id: hal-00541541

https://hal-mines-paristech.archives-ouvertes.fr/hal-00541541

Submitted on 30 Nov 2010

HAL is a multi-disciplinary open access archive for the deposit and dissemination of scientific research documents, whether they are published or not. The documents may come from teaching and research institutions in France or abroad, or from public or private research centers.
L'archive ouverte pluridisciplinaire HAL, est destinée au dépôt et à la diffusion de documents scientifiques de niveau recherche, publiés ou non, émanant des établissements d'enseignement et de recherche français ou étrangers, des laboratoires publics ou privés. 


\title{
Robust asymptotic stabilization of nonlinear systems by state feedback
}

\author{
François Poulain* Laurent Praly** \\ * ONERA-DPRS, BPr2 - FR-92322 Châtillon Cedex \\ (email: Francois.Poulain@onera.fr). \\ ** MINES ParisTech, CAS, Mathématiques et Systèmes, 35, rue \\ St-Honoré, 77305 Fontainebleau CEDEX, France \\ (email: Laurent.Praly@mines-paristech.fr).
}

\begin{abstract}
We show that regulation to a constant value of the output of a process can be achieved robustly by designing a stabilizer for a model augmented with an integrator of the output and by having the model dynamics close enough to the process ones. This is nothing but the PI controller paradigm extended to the case of nonlinear systems. We recall also that the forwarding technique is well suited for this particular stabilizer design. Finally we illustrate our result with solving the problem of regulating the flight path angle of the longitudinal mode of a plane.
\end{abstract}

Keywords: Asymptotic stability, forwarding, Integral action, nonlinear control, non-minimum phase systems, regulation, robust stability, uncertain dynamic system.

\section{INTRODUCTION}

Achieving a robust asymptotic regulation of a given output of a dynamical system is a question of practical interest in most industrial control design. We have identified two distinct families of designs in the literature.

In the first family, the design follows the same route as for linear systems. It consists in simply integrating ex nihilo the output error, and in stabilizing the extended system. The convergence of the output to its desired value is then a consequence of asymptotic stability. Various ways of designing the stabilizer for the extended system have been proposed. For instance Khalil (2000) obtains semiglobal robust stabilization by output feedback using high gain / sliding mode techniques.

In the second family, the dynamics are rewritten in such a way that an input perturbation, called steady state control, appears and that cancelling this term is sufficient to get the right invariant set. So part of the strategy consists in observing this shift to be applied.

The theory of output regulation can be seen in this perspective. Early results related to weakly nonlinear systems are due to Francis and Wonham (1976) where it was shown that integral control is sufficient to guarantee output regulation in presence of a constant exogenous input and small parameter variations. Latest results on nonlinear output regulation can obviously be applied to achieve robust asymptotic regulation, by considering an integrator as the internal model of the controller. For example, in Isidori et al. (2003) is described a complete framework to build output regulators for minimum phase systems.

Several other kind of input perturbation observers have been proposed. The works of Praly and Jiang (1998) and
Chakrabortty and Arcak (2007) introduce an observer of a dynamic input uncertainty, in order to reject it under still under a minimum phase assumption. In addition, Chakrabortty and Arcak (2007) need perfect knowledge of the input vector field to insure asymptotic regulation. In (Astolfi et al., 2008, Chapter 7.2), the authors solved a similar problem using dynamic controller, but discontinuous control law was used to stabilize the extended system, and robustness of the regulation has not been established.

Extension of the above when the term to be removed is not in the image of the control has been done using adaptive control techniques. Freeman and Kokotović (1996) do this for input/output linearizable systems with no zero dynamics. In Jiang and Mareels (2001), the authors allow the presence of inverse dynamics but with an ISS assumption.

After stating the problem, (Section 2), we present our proposed approach (Section 3). Next we recall forwarding tools specialized to our context of our interest (Section 4). Finally an example is given, inspired from a concrete case study in aeronautics (Section 5). Appendix A is devoted to demonstrate a technical result.

\section{PROBLEM STATEMENT}

We investigate the problem of regulating, by state feedback, the output $y$ of a given dynamical process to the origin. The considered process satisfies

$$
\dot{x}=\xi(x, u), \quad y=\zeta(x, u),
$$

where the state $x$ in $\mathbb{R}^{n}$, the control $u$ in $\mathbb{R}^{m}$ and the output $y$ in $\mathbb{R}^{m}$. The functions $\xi: \mathbb{R}^{n} \times \mathbb{R}^{m} \rightarrow \mathbb{R}^{n}$ and $\zeta: \mathbb{R}^{n} \times \mathbb{R}^{m} \rightarrow \mathbb{R}^{m}$ are assumed $C^{1}$.

We do not assume the knowledge of the process, i.e. of the pair $(\xi, \zeta)$. Precisely, we design the state feedback from a model of the process only given as 


$$
\dot{x}=f(x)+g(x) u, \quad y=h(x),
$$

where $f: \mathbb{R}^{n} \rightarrow \mathbb{R}^{n}, g: \mathbb{R}^{n} \rightarrow \mathbb{R}^{n m}$ and $h: \mathbb{R}^{n} \rightarrow \mathbb{R}^{m}$ are $C^{1}$ functions, and $f$ and $h$ vanish at origin.

The state $x$ is assumed to be measured and the output $y$ define $m$ signals which must converge to a constant reference chosen as 0 without loss of generality.

All along this document, process refers to the approximated dynamical process (1); whereas model means the known mathematical expression (2).

\section{SUGGESTED APPROACH}

To solve the problem of regulating $y$ to 0 in the uncertain context described above we follow the very classical idea of augmenting the model with a pure integrator ${ }^{1}$. Namely we design a state feedback stabilizing the origin of the system $^{2}$

$$
\dot{z}=k(x, y), \quad \dot{x}=f(x)+g(x) u,
$$

where $k: \mathbb{R}^{n} \times \mathbb{R}^{m} \rightarrow \mathbb{R}^{m}$ is a function to be chosen verifying

$$
\forall x \in \mathbb{R}^{n}, \quad k(x, h(x))=0 \quad \Rightarrow \quad h(x)=0 .
$$

Thanks to a "folk" result given in appendix we are able to conclude that the state feedback, designed for (3), solves the problem for a whole family of neighboring processes. Precisely we have

Proposition 1. Assume we have designed a function $\psi$ : $\mathbb{R}^{n} \times \mathbb{R}^{m} \rightarrow \mathbb{R}^{m}$ such that the origin is an exponentially stable equilibrium point of:

$$
\dot{z}=k(x, h(x)), \quad \dot{x}=f(x)+g(x) \psi(x, z),
$$

with $\mathcal{D} \times \mathbb{R}^{m}$ as domain of attraction. Under this condition, for any strictly positive real number $\varepsilon$, there exists a strictly positive real number $\delta$ and a neighborhood $\mathcal{N}$ of the origin such that, to any pair $(\xi, \zeta)$ of $C^{1}$ functions which satisfies:

$$
\begin{aligned}
& \mid k(x, h(x))-k(x, \zeta(x, \psi(x, z)) \mid \\
& \quad+|[f(x)+g(x) \psi(x, z)]-\xi(x, \psi(x, z))| \leq \delta,
\end{aligned}
$$

for all $(x, z)$ in $\mathcal{D} \times \mathbb{R}^{m}$ and

$$
\begin{aligned}
& \mid\left(\begin{array}{cc}
0 & \frac{\partial k}{\partial x}+\frac{\partial k}{\partial y} \frac{\partial h}{\partial x} \\
g \frac{\partial \psi}{\partial z} & \frac{\partial f}{\partial x}+\frac{\partial g}{\partial x} \psi+g \frac{\partial \psi}{\partial x}
\end{array}\right) \\
& -\left(\begin{array}{cc}
\frac{\partial k}{\partial y} \frac{\partial \zeta}{\partial u} \frac{\partial \psi}{\partial z} & \frac{\partial k}{\partial x}+\frac{\partial k}{\partial y}\left[\frac{\partial \zeta}{\partial x}+\frac{\partial \zeta}{\partial u} \frac{\partial \psi}{\partial z}\right] \\
\frac{\partial \xi}{\partial u} \frac{\partial \psi}{\partial z} & \frac{\partial \xi}{\partial x}+\frac{\partial \xi}{\partial u} \frac{\partial \psi}{\partial x}
\end{array}\right) \mid \leq \delta,
\end{aligned}
$$

for all $(z, x)$ in $\mathcal{N}$, we can associate $\left(x_{e}, z_{e}\right) \in \mathcal{N}$ which is an exponentially stable equilibrium point of:

$$
\dot{z}=k(x, \zeta(x, \psi(x, z))), \quad \dot{x}=\xi(x, \psi(x, z)),
$$

whose basin of attraction contains the set

$$
\mathcal{A}=\left\{(x, z) \in \mathcal{D} \times \mathbb{R}^{m}: d\left((x, z), \partial\left(\mathcal{D} \times \mathbb{R}^{m}\right)\right)>\varepsilon\right\} .
$$

Also, under the above condition and (4), any solution $(X((x, z), t), Z((x, z), t))$ of $(8)$ with initial condition in the above set satisfies:

$$
\lim _{t \rightarrow+\infty} \zeta(X((x, z), t), \psi(X((x, z), t), Z((x, z), t)))=0 .
$$

1 See (Khalil, 2002, page 481) for instance for an explanation.

2 The interest of replacing $h$ by $k$ will be illustrated in Section 5 .
Proof. With the notations

$$
\begin{gathered}
\mathcal{X}=\left(\begin{array}{c}
z \\
x
\end{array}\right), \\
\hat{\varphi}(\mathcal{X})=\left(\begin{array}{c}
k(x, h(x)) \\
f(x)+g(x) u
\end{array}\right), \quad \varphi(\mathcal{X})=\left(\begin{array}{c}
k(x, \zeta(x, u)) \\
\xi(x, u)
\end{array}\right),
\end{gathered}
$$

we see that all the assumptions of Theorem 6 are satisfied. It follows that if the pair of functions $(\xi, \zeta)$ satisfies $(6)$ and (7), we have an asymptotically stable equilibrium point $\left(x_{e}, z_{e}\right)$ with $\mathcal{A}$ given in (9) as basin of attraction and which satisfies

$$
k\left(x_{e}, \zeta\left(x_{e}, \psi\left(x_{e}, z_{e}\right)\right)\right)=0 .
$$

But (4) and continuity imply the existence of a class $\mathcal{K}^{\infty}$ function $\rho$ such that we have:

$$
|y| \leq \rho(|k(x, y)|)[1+\rho(|x|+|y|)] \quad \forall(x, y) .
$$

Hence we have that, for any initial condition $(x, z)$ in $\mathcal{A}$, the corresponding solution $(X((x, z), t), Z((x, z), t))$ of $(8)$ is defined on $[0,+\infty)$, converges to $\left(x_{e}, z_{e}\right)$ and satisfies (10).

Remark 2. Equation (11) is nothing but a regulator equation, since $\left(x_{e}, z_{e}\right)$ is an equilibrium of (8).

\section{DESIGN OF THE FEEDBACK $\psi$}

In this section we discuss possible feedback designs which guarantee the assumption about system (5) in Proposition 1 is met.

The extended system (3) has one of the block-triangular forms that has been extensively studied in the 90's with in particular the introduction of the techniques of backstepping (see Krstic et al. (1995) for an overview) and forwarding. The latter has been developed using saturations in Teel (1996) or using Lyapunov design with coordinate change in Mazenc and Praly (1996) or coupling term in Jankovic et al. (1996). Here we recall these forwarding techniques. One of their interesting features is the availability to simply "extend" an existing static controller which stabilize the origin of (2). In the following, we call $\phi(x)$ this controller.

Assumption 3. (Stability of statically controlled system). We know a continuous function $\phi: \mathbb{R}^{n} \rightarrow \mathbb{R}^{m}$ such that the origin is an asymptotically and locally exponentially stable equilibrium point with $\mathcal{D}$ as domain of attraction.

As a consequence of this assumption, using converse Lyapunov theorem from Kurzweil (1956), there exists a $C^{1}$ function $V: \mathcal{D} \rightarrow \mathbb{R}^{+}$which is positive definite and proper on $\mathcal{D}$ such that the function $U$ defined below is positive definite on $\mathcal{D}$.

$$
U(x)=-\frac{\partial V}{\partial x}(f(x)+g(x) \phi(x))
$$

Depending on whether or not we know such a function $V$ leads to different designs.

Assumption 4. (Rank of static gain). The matrix

$$
\left.\left(\frac{\partial k}{\partial x}+\frac{\partial k}{\partial y} \frac{\partial h}{\partial x}\right)\left(\frac{\partial}{\partial x}[f+g \phi]\right)^{-1} g\right|_{x=0}
$$

is invertible. 
A consequence of Assumption 3 is the existence of a $C^{1}$ function $M: \mathcal{D} \rightarrow \mathbb{R}^{m}$ satisfying

$$
\frac{\partial M}{\partial x}(x)(f(x)+g(x) \phi(x))=k(x, h(x)), \quad M(0)=0 .
$$

Depending on whether or not we know this function or only its first order approximation at the origin gives different designs.

\subsection{Forwarding with $V$ and $M$ known}

When both $V$ and $M$ are known a stabilizer for the system (3) is

$$
u=\phi(x)-L\left(\left[L_{g} V(x)-(z-M(x))^{\top} L_{g} M(x)\right]^{\top}\right),
$$

with $M$ defined by (15), and with $L$ any continuous function satisfying

$$
v^{\top} L(v)>0, \quad \forall v \neq 0, \quad \operatorname{det}\left(\frac{\partial L}{\partial v}(0)\right) \neq 0 .
$$

Following Mazenc and Praly (1996) this can be established with the function $W: \mathcal{D} \times \mathbb{R}^{m} \rightarrow \mathbb{R}_{+}$defined as

$$
W(x, z)=V(x)+\frac{1}{2}(z-M(x))^{\top}(z-M(x)) .
$$

which is positive definite and proper on $\mathcal{D} \times \mathbb{R}^{m}$.

\subsection{Forwarding with $V$ unknown but $M$ known.}

When $V$ is unknown, but $M$ is known, there exists a scalar function $k_{i}: \mathcal{D} \rightarrow \mathbb{R}+$ such that a state feedback for the system (3) is

$$
u=\phi(x)+k_{i}(x) L_{g} M(x)^{\top} L(z-M(x)),
$$

with $M$ defined by (15), and with $L$ any bounded continuous function satisfying (17).

This can be established with the Lyapunov function (18).

\subsection{Forwarding with $V$ unknown and $M$ approximated.}

Instead of solving the partial differential equation (15) for $M$, we can simply solve the linear equation

$$
\left.M_{0} \frac{\partial}{\partial x}[f(x)+g(x) \phi(x)]\right|_{x=0}=\frac{\partial}{\partial x}\left(\left.k(x, h(x))\right|_{x=0} .\right.
$$

This can always be done, thanks to Assumption 4.

In this case, there exists a function $k_{i}: \mathcal{D} \rightarrow \mathbb{R}+$ with strictly positive values such that a state feedback for the system (3) is

$$
u=\phi(x)+k_{i}(x) M_{0}^{\top} g(x)^{\top} L\left(z-M_{0} x\right),
$$

with $M_{0}$ defined by (20), and with $L$ any bounded continuous satisfying (17).

This can be established with the Lyapunov function

$$
W(x, z)=e(V(x))+\sqrt{1+\frac{1}{2}\left(z-M_{0} x\right)^{\top}\left(z-M_{0} x\right)}-1,
$$

where $e: \mathbb{R}^{+} \rightarrow \mathbb{R}^{+}$is a $C^{1}$ function with strictly positive derivative, to be chosen large enough (see Mazenc and Praly (1996)).
In the case where the system

$$
\dot{x}=f(x)+g(x)(\phi(x)+v),
$$

with $v$ as input is input to state stable with restriction, i.e. provided $|v|$ is bounded by some given strictly positive real number $\Delta$, then following Teel (1996), the state feedback can be chosen as

$$
u=\phi(x)+\lambda L\left(\frac{g(0)^{\top} M_{0}^{\top}\left(z-M_{0} x\right)}{\lambda}\right),
$$

where $L$ is any bounded continuous function satisfying (17) and $\lambda$ is a small enough strictly positive real number.

Remark 5. (Link with an observer point of view). The approach proposed above for the case where $V$ is unknown can also be seen as a perturbation observer. To this end, consider the case where there is a constant input perturbation $\delta$ in the model (2), i.e. its dynamics are

$$
\dot{x}=f(x)+g(x)(u+\delta), \quad \dot{\delta}=0 .
$$

Assume there exist a $C^{1}$ function $K$, a square matrix $N$ and a positive definite matrix $P$ satisfying

$$
P N L_{g} K(x)+\left[N L_{g} K(x)\right]^{\top} P<0, \quad \forall x \in \mathcal{D} .
$$

Note this condition is met for $x$ close to the origin under Assumption 4 if we pick

$$
K(x)=M(x), \quad N=-L_{g} M(0)^{\top} .
$$

Then a reduced order observer for $\delta$ is given by

$$
\begin{aligned}
& \dot{z}=\left[L_{f} K(x)+L_{g} K(x) u\right]+L_{g} K(x) N[z-K(x)], \\
& \widehat{\delta}=N[z-K(x)] .
\end{aligned}
$$

Indeed, we have

$$
\overbrace{(\widehat{\delta}-\delta)^{\top} P(\widehat{\delta}-\delta)}<0 .
$$

Cancelling $\delta$ with the feedback leads to the dynamic feedback

$$
\dot{z}=L_{f} K(x)+L_{g} K(x) \phi(x), \quad u=\phi(x)-N[z-K(x)] .
$$

So we do recover (19) if we pick

$$
\begin{aligned}
K(x) & =M(x), \\
L(z-M(x)) & =-(z-M(x)), \\
N & =-k_{i}(x) L_{g} M(x)^{\top} .
\end{aligned}
$$

This link with a reduced order observer brings some more light on the role played by $k_{i}$. In particular we understand that the value at $x$ for $k_{i}(x)$ should be such that either it is zero or $k_{i}(x) L_{g} M(x)^{\top}$ is close to $k_{i}(0) L_{g} M(0)^{\top}$ and we have

$$
P L_{g} M(0)^{\top} L_{g} M(x)+\left[L_{g} M(0)^{\top} L_{g} M(x)\right]^{\top} P>0 .
$$

\section{EXAMPLES OF APPLICATION: THE LONGITUDINAL MODE OF A PLANE}

A very elementary approximation of the flight dynamics of an aerodyne is

$$
\dot{V}=e-\sin \gamma, \quad \dot{\gamma}=\frac{l V^{2}-\cos \gamma}{V},
$$

where $V \in \mathbb{R}_{+}^{*}$ is the speed and $\gamma \in \mathbb{R}$ is the flight path angle. The controls are the lift $l$ and the propulsive balance $e$. This is an oscillator early studied by Zhukovskii in the XIXe century (see Andronov et al. (1987)). It has stable periodic solutions for fixed values $e=0$ and $l=1 / V_{0}^{2}>0$. Indeed, it can be easily verified that the 
equilibrium $\left(\gamma=0, V_{0}\right)$ is stable, by the use of the energy function

$$
W(V, \gamma)=V^{3}+2 V_{0}^{3}-3 V V_{0}^{2} \cos (\gamma),
$$

which is non negative for all $(V, \gamma) \in \mathbb{R}^{+} \times[-\pi ; \pi]$ and vanishes only when $(V, \gamma)=\left(V_{0}, 0\right)$ but whose sublevel sets do not contain points with $V \leq 0$ only for $W(V, \gamma)<2 V_{0}^{3}$. This stability property can be exploited to design the following controller

$$
l=\frac{1}{V_{0}^{2}}-\frac{V^{2}}{V_{0}^{4}} \sin \gamma, \quad e=\cos \gamma-\left(\frac{V}{V_{0}}\right)^{2} .
$$

which makes the equilibrium $\left(V_{0}, 0\right)$ exponentially stable with domain of attraction containing the set $\{(V, \gamma): V>$ $\left.0, W(V, \gamma)<2 V_{0}^{3}\right\}$. See Poulain et al. (2009).

The longitudinal mode model (28) of the vehicle being only an approximation, the stabilized equilibrium obtained with the controller (30) for this vehicle, even if it exists, is likely to differ from the desired one. Insisting on the convergence of the flight path angle to its desired value we choose it as output. In order to apply the design of Section 4.2 , we have then to find functions $M$ and $k$ verifying

$$
\frac{\partial M}{\partial V}(e-\sin \gamma)+\frac{\partial M}{\partial \gamma} \frac{\left(1-\frac{V^{2}}{V_{0}^{2}} \sin \gamma\right) \frac{V^{2}}{V_{0}^{2}}-\cos \gamma}{V}=k,
$$

and

$$
k(V, \gamma)=0 \Rightarrow \gamma=0 .
$$

We observe that choosing

$$
k(V, \gamma)=-\left(\frac{\partial M}{\partial V}+\frac{\partial M}{\partial \gamma} V^{3}\right) \sin \gamma+e(1-\cos \gamma) \frac{\partial M}{\partial V},
$$

which satisfies (32) in the domain of interest, the partial differential equation (31) reduces to

$$
\frac{\partial M}{\partial V} e \cos \gamma-\frac{\partial M}{\partial \gamma} \frac{e}{V}=0
$$

for which

$$
M(V, \gamma)=\ln (V)+\sin (\gamma)-\ln \left(V_{0}\right)
$$

is a solution. Also, fortunately, with this $k$ the model controlled with (30) verifies the static gain requirement.

The controller, extended with an integral action thus becomes

$$
\begin{aligned}
& \dot{z}=-\left(\frac{1}{V}+\frac{V^{3}}{V_{0}^{4}} \cos \gamma\right) \sin \gamma+\frac{1}{V}(1-\cos \gamma) e \\
& e=\cos \gamma-\left(\frac{V}{V_{0}}\right)^{2} \\
& l=l_{0}-V^{2} \sin \gamma+z-\left(\ln (V)+\sin (\gamma)-\ln \left(V_{0}\right)\right)
\end{aligned}
$$

It guarantees asymptotic regulation of the output on the process provided it is close enough to the model (28).

The simulation results are presented on Figure 1, where no special gain optimization was done. The process is taken as

$$
\begin{aligned}
\varepsilon \dot{\hat{l}} & =l-\hat{l}, \\
\dot{V} & =e \cos \alpha-\sin \gamma+(\cos \alpha-\epsilon) \hat{l}^{2} V^{2}, \\
V \dot{\gamma} & =\epsilon \hat{l} V^{2}-\cos \gamma+e \sin \alpha,
\end{aligned}
$$

with $\alpha=\arcsin (\hat{l} / \mu)+\nu, \varepsilon=0.33, \epsilon=0.6, \nu=$ 0.1 and $\mu=5$. These coefficients are representative of modelling simplifications or uncertainties. Aerodynamics
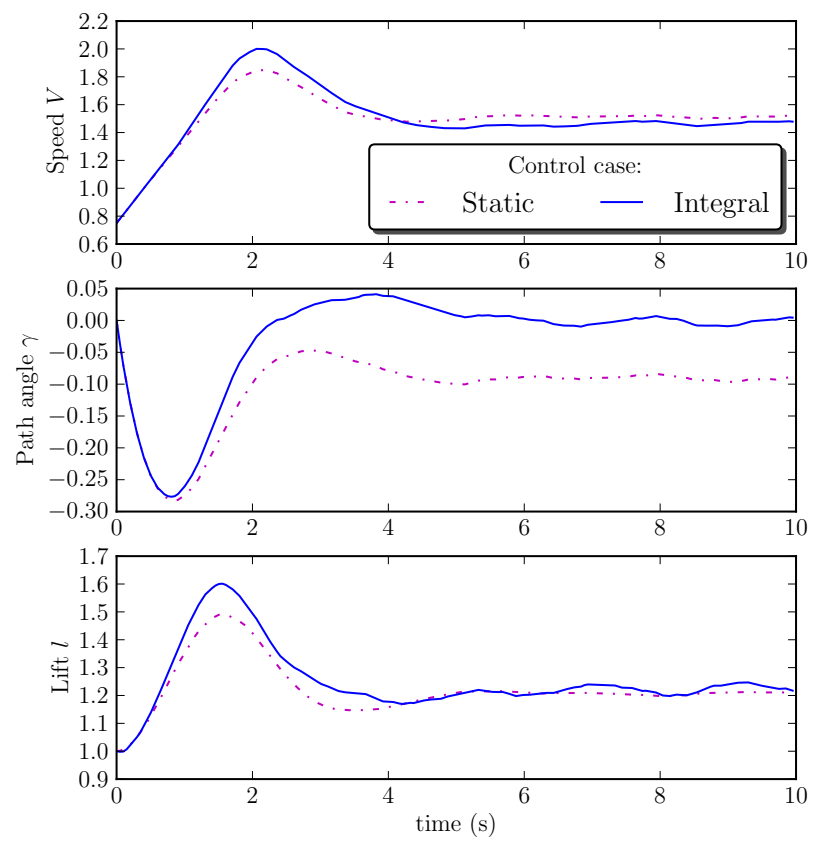

Fig. 1. Simulation of the Zhukovskii oscillator in perturbed conditions (i) without integral action (dash-dotted line); (ii) with an integral action (solid line).

are then scaled by $\epsilon$, and the thrust direction of the vehicle, assumed to be in the speed direction, is oriented in the process by $\alpha$. Furthermore, filtered measurement noise and input dynamic are added. Asymptotic behavior of the flight path angle is then clearly better using the controller (35) using integral action compared with (30) with no integral action.

\section{CONCLUSION}

Robust asymptotic output regulation has been investigated. Our technique follows the very usual approach of stabilizing the origin of the model augmented with an integrator of the output error. The result is shown to be robust to any small enough perturbation of the model, in a $C^{1}$ sense.

We use forwarding as a tool to design state feedback. This allows us to handle models with no specific structure but for which we have already a stabilizer. These systems can be non-minimum phase. The basic required property is the invertibility of the static gain at the origin, the standard "non resonance" condition.

Finally, we observe that it is possible to extend what we have presented to the context of output feedback by following one approach of Teel and Praly (1994). The details of this extension are under investigation.

\section{REFERENCES}

Andronov, A.A., Vitt, A.A., and Khaikin, S.E. (1987). Theory of Oscillators. Dover.

Astolfi, A., Karagiannis, D., and Ortega, R. (2008). Nonlinear and Adaptive Control with Applications. Communications and Control Engineering. Springer. 
Chakrabortty, A. and Arcak, M. (2007). A Two-TimeScale Redesign for Robust Stabilization and Performance Recovery of Uncertain Nonlinear Systems. American Control Conference, 200\%. ACC '07, 4643-4648.

Francis, B. and Wonham, W. (1976). The internal model principle of control theory. Automatica, 12(5), 457-465.

Freeman, R.A. and Kokotović, P.V. (1996). Robust Nonlinear Control Design. Modern Birkhuser Classics. Birkhäuser.

Hale, J.K. (1980). Ordinary Differential Equations. Krieger Publishing Company.

Isidori, A., Marconi, L., and Serrani, A. (2003). Robust Autonomous Guidance. Advances in industrial control. Springer.

Jankovic, M., Sepulchre, R., and Kokotovic, P. (1996). Constructive lyapunov stabilization of nonlinear cascade systems. Automatic Control, IEEE Transactions on, 41(12), 1723-1735.

Jiang, Z.P. and Mareels, I. (2001). Robust nonlinear integral control. Automatic Control, IEEE Transactions on, 46(8), 1336-1342.

Khalil, H.K. (2000). Universal integral controllers for minimum-phase nonlinear systems. Automatic Control, IEEE Transactions on, 45(3), 490-494.

Khalil, H.K. (2002). Nonlinear Systems. Prentice Hall, third edition.

Krstic, M., Kanellakopoulos, I., and Kokotovic, P. (1995). Nonlinear and Adaptive Control Design. John Wiley and Sons.

Kurzweil, J. (1956). On the inversion of lyapunov's second theorem on stability of motion. Amer. Math. Soc. Transl. Ser., 24(2), 19-77.

Mazenc, F. and Praly, L. (1996). Adding integrations, saturated controls, and stabilization for feedforward systems. Automatic Control, IEEE Transactions on, 41(11), 1559-1578.

Poulain, F., Piet-Lahanier, H., and Serre, L. (2009). Nonlinear control of a airbreathing hypersonic vehicle. In 16th $A I A A / D L R / D G L R$ International Space Planes and Hypersonic Systems and Technologies Conference. Bremen, Germany.

Praly, L. and Jiang, Z.P. (1998). Semiglobal stabilization in the presence of minimum-phase dynamic input uncertainties. In NOLCOS'98, volume 2, 325-330.

Teel, A. and Praly, L. (1994). Global stabilizability and observability imply semi-global stabilizability by output feedback. Systems \& Control Letters, 22(5), 313-325.

Teel, A. (1996). A nonlinear small gain theorem for the analysis of control systems with saturation. Automatic Control, IEEE Transactions on, 41(9), 1256-1270.

\section{Appendix A. A TOTAL STABILITY THEOREM}

The following result combines total stability and hyperbolicity.

Theorem 6 . Let be given a $C^{1}$ function $\hat{\varphi}: \mathbb{R}^{n} \rightarrow \mathbb{R}^{n}$ such that the origin is an exponentially stable equilibrium point of:

$$
\dot{\mathcal{X}}=\hat{\varphi}(\mathcal{X})
$$

with $\mathcal{A}$ as domain of attraction. Under this condition, for any strictly positive real number $\varepsilon$, there exists a strictly positive real number $\delta$ and a neighborhood $\mathcal{N}$ of the origin such that, to any $C^{1}$ function $\varphi: \mathbb{R}^{n} \rightarrow \mathbb{R}^{n}$ which satisfies:

$$
\begin{aligned}
|\varphi(\mathcal{X})-\hat{\varphi}(\mathcal{X})| & \leq \delta, & \forall \mathcal{X} \in \mathcal{A}, \\
\left|\frac{\partial \varphi}{\partial \mathcal{X}}(\mathcal{X})-\frac{\partial \hat{\varphi}}{\partial \mathcal{X}}(\mathcal{X})\right| & \leq \delta, & \forall \mathcal{X} \in \mathcal{N},
\end{aligned}
$$

we can associate $\mathcal{X}_{e}$ which is an exponentially stable equilibrium point of:

$$
\dot{\mathcal{X}}=\varphi(\mathcal{X})
$$

whose basin of attraction contains the set

$$
\{\mathcal{X} \in \mathcal{A}: d(\mathcal{X}, \partial \mathcal{A})>\varepsilon\} .
$$

This is a "folk" result. But being unable at this time to give a reference containing a complete proof and to make the paper self-contained, we give a proof below which has some similarities with (Khalil, 2002, Section 14.1.4).

Proof. Let $P$ be a positive definite symmetric matrix and $a$ a strictly positive real number satisfying:

$$
P \frac{\partial \hat{\varphi}}{\partial \mathcal{X}}(0)+\frac{\partial \hat{\varphi}}{\partial \mathcal{X}}(0)^{\top} P \leq-a P, \quad \underline{\lambda}(P)=1,
$$

where $\bar{\lambda}$ and $\underline{\lambda}$ respectively stand for max and min singular values. By continuity there exists a strictly positive real number $p_{0}$ such that we have, $\forall \mathcal{X}: \mathcal{X}^{\top} P \mathcal{X} \leq p_{0}$,

$P \frac{\partial \hat{\varphi}}{\partial \mathcal{X}}(\mathcal{X})+\frac{\partial \hat{\varphi}}{\partial \mathcal{X}}(\mathcal{X})^{\top} P \leq-\frac{a}{2} P$, and $\mathcal{X}^{\top} P \hat{\varphi}(\mathcal{X}) \leq-\frac{a}{4} \mathcal{X}^{\top} P \mathcal{X}$.

Let $\varphi: \mathbb{R}^{n} \rightarrow \mathbb{R}^{n}$ be a $C^{1}$ function. We have:

$$
\begin{aligned}
\mathcal{X}^{\top} P \varphi(\mathcal{X})= & \mathcal{X}^{\top} P \hat{\varphi}(\mathcal{X})+\mathcal{X}^{\top} P[\varphi(\mathcal{X})-\hat{\varphi}(\mathcal{X})], \\
\leq & \mathcal{X}^{\top} P \hat{\varphi}(\mathcal{X})+\frac{a}{8} \mathcal{X}^{\top} P \mathcal{X} \\
& +\frac{2}{a}[\varphi(\mathcal{X})-\hat{\varphi}(\mathcal{X})]^{\top} P[\varphi(\mathcal{X})-\hat{\varphi}(\mathcal{X})]
\end{aligned}
$$

and therefore:

$$
\mathcal{X}^{\top} P \varphi(\mathcal{X}) \leq-\frac{a}{16} \mathcal{X}^{\top} P \mathcal{X}, \quad \forall \mathcal{X}: \mathcal{X}^{\top} P \mathcal{X}=\frac{p_{0}}{6},
$$

provided $\varphi$ satisfies:

$$
|\varphi(\mathcal{X})-\hat{\varphi}(\mathcal{X})| \leq \frac{a}{4} \sqrt{\frac{p_{0}}{12 \bar{\lambda}(P)}}, \quad \forall \mathcal{X}: \mathcal{X}^{\top} P \mathcal{X}=\frac{p_{0}}{6} .
$$

In this condition, it follows from Brouwer fixed point Theorem (See (Hale, 1980, Theorem 8.2)) that, for each function $\varphi$ satisfying (A.10), there exits a point $\mathcal{X}_{e}$ satisfying:

$$
\varphi\left(\mathcal{X}_{e}\right)=0, \quad \mathcal{X}_{e}^{\top} P \mathcal{X}_{e} \leq \frac{p_{0}}{6} .
$$

Assume further that $\varphi$ satisfies:

$$
\left|\frac{\partial \varphi}{\partial \mathcal{X}}(\mathcal{X})-\frac{\partial \hat{\varphi}}{\partial \mathcal{X}}(\mathcal{X})\right| \leq \frac{a}{8 \bar{\lambda}(P)}, \quad \forall \mathcal{X}: \mathcal{X}^{\top} P \mathcal{X} \leq p_{0} .
$$

In this case, we have:

$$
\begin{aligned}
& P \frac{\partial \varphi}{\partial \mathcal{X}}(\mathcal{X})+\frac{\partial \varphi}{\partial \mathcal{X}}(\mathcal{X})^{\top} P= \\
& P \frac{\partial \hat{\varphi}}{\partial \mathcal{X}}(\mathcal{X})+\frac{\partial \hat{\varphi}}{\partial \mathcal{X}}(\mathcal{X})^{\top} P+P\left[\frac{\partial \varphi}{\partial \mathcal{X}}(\mathcal{X})-\frac{\partial \hat{\varphi}}{\partial \mathcal{X}}(\mathcal{X})\right] \\
&+\left[\frac{\partial \varphi}{\partial \mathcal{X}}(\mathcal{X})-\frac{\partial \hat{\varphi}}{\partial \mathcal{X}}(\mathcal{X})\right]^{\top} P \\
& \leq-\frac{a}{4} P, \quad \forall \mathcal{X}: \mathcal{X}^{\top} P \mathcal{X} \leq p_{0} .
\end{aligned}
$$

Note also that we have:

$$
\left[\mathcal{X}_{e}+s\left(\mathcal{X}-\mathcal{X}_{e}\right)\right]^{\top} P\left[\mathcal{X}_{e}+s\left(\mathcal{X}-\mathcal{X}_{e}\right)\right] \leq p_{0},
$$




$$
\forall\left(\mathcal{X}, \mathcal{X}_{e}, s\right): s \in[0,1], \quad \mathcal{X}_{e}^{\top} P \mathcal{X}_{e} \leq \frac{p_{0}}{6}, \quad \mathcal{X}^{\top} P \mathcal{X} \leq \frac{p_{0}}{3} .
$$

Then, with

$\varphi(\mathcal{X})=\varphi(\mathcal{X})-\varphi\left(\mathcal{X}_{e}\right)=\int_{0}^{1} \frac{\partial \varphi}{\partial \mathcal{X}}\left(\mathcal{X}_{e}+s\left(\mathcal{X}-\mathcal{X}_{e}\right)\right) d s\left[\mathcal{X}-\mathcal{X}_{e}\right]$,

and (A.11), we get:

$$
\begin{aligned}
& {\left[\mathcal{X}-\mathcal{X}_{e}\right]^{\top} P \varphi(\mathcal{X})=} \\
& \int_{0}^{1}\left(\left[\mathcal{X}-\mathcal{X}_{e}\right]^{\top} P \frac{\partial \varphi}{\partial \mathcal{X}}\left(\mathcal{X}_{e}+s\left(\mathcal{X}-\mathcal{X}_{e}\right)\right)\left[\mathcal{X}-\mathcal{X}_{e}\right]\right) d s \\
& \quad \leq-\frac{a}{4}\left[\mathcal{X}-\mathcal{X}_{e}\right]^{\top} P\left[\mathcal{X}-\mathcal{X}_{e}\right], \quad \forall \mathcal{X}: \mathcal{X}^{\top} P \mathcal{X} \leq \frac{p_{0}}{3} .
\end{aligned}
$$

This implies that $\mathcal{X}_{e}$ is an exponentially stable equilibrium point of the system (A.4) with basin of attraction containing the compact set $\left\{\mathcal{X} \in \mathbb{R}^{n}: \mathcal{X}^{\top} P \mathcal{X} \leq \frac{p_{0}}{3}\right\}$.

Finally, from the assumption on $\hat{\varphi}$, we know there exists a function $V: \mathcal{A} \rightarrow R_{+}$which is $C^{1}$, positive definite and satisfies:

$$
\lim _{\mathcal{X} \rightarrow \partial \mathcal{A}} V(\mathcal{X})=+\infty, \quad \frac{\partial V}{\partial \mathcal{X}} \hat{\varphi}(\mathcal{X}) \leq-V(\mathcal{X}), \quad \forall \mathcal{X} \in \mathcal{A} .
$$

Then let $v_{0}$ and $v_{\infty}$ be strictly positive real numbers such that we have:

$$
\begin{array}{rlrl}
v_{0} & <v_{\infty}, & & \\
\mathcal{X}^{\top} P \mathcal{X} & \leq \frac{p_{0}}{3}, & & \forall \mathcal{X} \in \mathcal{A}: V(\mathcal{X}) \leq v_{0} \\
d(\mathcal{X}, \partial \mathcal{A}) & \leq \varepsilon, & \forall \mathcal{X} \in \mathcal{A}: V(\mathcal{X}) \geq v_{\infty} .
\end{array}
$$

With (A.13) and since the set

$$
C=\left\{\mathcal{X} \in \mathcal{A}: v_{0} \leq V(\mathcal{X}) \leq v_{\infty}\right\}
$$

is a compact subset of $\mathcal{A}$ where $V$ is $C^{1}$, the real number obtained as:

$$
G=\sup _{\mathcal{X} \in C}\left|\frac{\partial V}{\partial \mathcal{X}}(\mathcal{X})\right|
$$

is well defined and strictly positive. We get:

$$
\begin{aligned}
\frac{\partial V}{\partial \mathcal{X}}(\mathcal{X}) \varphi(\mathcal{X}) & =\frac{\partial V}{\partial \mathcal{X}}(\mathcal{X}) \hat{\varphi}(\mathcal{X})+\frac{\partial V}{\partial \mathcal{X}}(\mathcal{X})[\varphi(\mathcal{X})-\hat{\varphi}(\mathcal{X})] \\
& \leq-V(\mathcal{X})+G \sup _{\mathcal{X} \in C}|\varphi(\mathcal{X})-\hat{\varphi}(\mathcal{X})|, \forall \mathcal{X} \in C .
\end{aligned}
$$

So if $\varphi$ satisfies also:

$$
\begin{aligned}
\forall \mathcal{X} \in \mathcal{A},|\varphi(\mathcal{X})-\hat{\varphi}(\mathcal{X})| \\
\quad \leq \frac{\inf _{\mathcal{X} \in C} V(\mathcal{X})}{2 G}=\frac{\inf _{\mathcal{X} \in C} V(\mathcal{X})}{2 \sup _{\mathcal{X} \in C}\left|\frac{\partial V}{\partial \mathcal{X}}(\mathcal{X})\right|},
\end{aligned}
$$

we have:

$$
\overparen{V(\mathcal{X})} \leq-\frac{1}{2} V(\mathcal{X}) \quad \forall \mathcal{X} \in C .
$$

This implies that the compact set $\left\{\mathcal{X} \in \mathcal{A}: V(\mathcal{X}) \leq v_{0}\right\}$ is asymptotically stable for the system (A.4) with basin of attraction containing the compact set defined as $\{\mathcal{X} \in \mathcal{A}$ : $\left.V(\mathcal{X}) \leq v_{\infty}\right\}$. But from (A.15), we have:

$\left\{\mathcal{X} \in \mathcal{A}: V(\mathcal{X}) \leq v_{0}\right\} \subset\left\{\mathcal{X} \in \mathbb{R}^{n}: \mathcal{X}^{\top} P \mathcal{X} \leq \frac{p_{0}}{3}\right\}$. (A.21) So, with (A.10), (A.12), (A.16), and (A.19) we have established our result with $\delta$ given as:

$$
\delta=\min \left\{\frac{a}{4} \sqrt{\frac{p_{0}}{12 \bar{\lambda}(P)}}, \frac{a}{8 \bar{\lambda}(P)}, \frac{\inf _{\mathcal{X} \in C} V(\mathcal{X})}{2 \sup _{\mathcal{X} \in C}\left|\frac{\partial V}{\partial \mathcal{X}}(\mathcal{X})\right|}\right\}
$$

and $\mathcal{N}$ given as:

$$
\mathcal{N}=\left\{\mathcal{X} \in \mathcal{A}: \mathcal{X}^{\top} P \mathcal{X} \leq p_{0}\right\} .
$$

